\title{
Landau-Khalatnikov-Fradkin transformation of the fermion propagator in massless reduced QED
}

\author{
A. James, ${ }^{1}$ A. V. Kotikov, ${ }^{2}$ and S. Teber ${ }^{1}$ \\ ${ }^{1}$ Sorbonne Université, Centre National de la Recherche Scientifique, \\ Laboratoire de Physique Théorique et Hautes Energies, LPTHE, F-75005 Paris, France \\ ${ }^{2}$ Bogoliubov Laboratory of Theoretical Physics, Joint Institute for Nuclear Research, \\ 141980 Dubna, Russia
}

(Received 19 December 2019; accepted 22 January 2020; published 18 February 2020)

\begin{abstract}
We study the gauge-covariance of the massless fermion propagator in reduced quantum electrodynamics (QED). Starting from its value in some gauge, we evaluate an all order expression for it in another gauge by means of the Landau-Khalatnikov-Fradkin transformation. We find that the weak-coupling expansions thus derived are in perfect agreement with the exact calculations. We also prove that the fermion anomalous dimension of reduced QED is gauge invariant to all orders of perturbation theory except for the first one.
\end{abstract}

DOI: 10.1103/PhysRevD.101.045011

\section{INTRODUCTION}

The Landau-Khalatnikov-Fradkin (LKF) transformation [1] (see also [2,3]) is an elegant and powerful transformation allowing one to study the gauge-covariance of Green's functions in gauge theories. In the latter, gauge freedom is implemented by a covariant gauge fixing procedure that introduces an explicit dependence of Green's functions on a gauge fixing parameter $\xi$. The LKF transformation then relates the Green's functions in two different $\xi$ gauges. Of course, physical quantities should not depend on $\xi$. But important information can be obtained by studying the $\xi$ dependence of various correlation functions.

In its original form, the LKF transformation was applied to the fermion propagator (and also to the fermion-photon vertex that will not be discussed here) of four-dimensional quantum electrodynamics $\left(\mathrm{QED}_{4}\right)$, which is the primary example of an Abelian gauge field theory. Since then, it has been extensively used in studies of QED in various dimensions, see, e.g., [4-11] and, more recently, in their generalization to brane worlds [12] that we shall come back to in the following and also to non-Abelian $S U(N)$ gauge field theories [13,14].

As a well-known application, let us first mention its crucial role within the study of QED Schwinger-Dyson equations; see, e.g., [4-6], where any viable charged-particle-photon vertex ansatz has to satisfy the LKF transformation, both for scalar [7] and spinor QED [8]. Another notable application

Published by the American Physical Society under the terms of the Creative Commons Attribution 4.0 International license. Further distribution of this work must maintain attribution to the author(s) and the published article's title, journal citation, and DOI. Funded by SCOAP ${ }^{3}$.
$[9,10]$ that will be closer to our present concerns is devoted to estimating the large order behavior of perturbative expansions. Namely, the nonperturbative nature of the LKF transformation fixes certain coefficients appearing in the all-order expansion of the fermion propagator. Given a perturbative propagator written for some fixed gauge parameter, say $\eta$, all the coefficients depending on the difference between the gauge fixing parameters of the two propagators, i.e., $\xi-\eta$, get fixed by the weak-coupling expansion of the LKF-transformed initial propagator. Recently, such a procedure allowed to prove [11] the so-called "no- $\pi$ theorem" [15-19], e.g., cancellations involving $\zeta_{2 n}$ (or equivalently $\pi^{2 n}$ ) values in the perturbative expansion of Euclidean fermion propagator in massless $\mathrm{QED}_{4}$, thereby clarifying the transcendental structure of the latter.

In the present paper, we apply the LKF transformation to the fermion propagator of massless reduced QED or $\mathrm{RQED}_{d_{y}, d_{\mathrm{e}}}$; see Refs. [20-22] and references therein. The latter is an Abelian gauge theory where the photon and fermion fields live in different space-time dimensionalities, namely, the photon is in $d_{\gamma}$ dimensions whereas the fermion fields are confined to $d_{e}$ dimensions, where we take $d_{e} \leq d_{\gamma}$. We shall focus on the special case of $\mathrm{RQED}_{4,3}$ which is an effective field theory for the so-called planar Dirac liquids, i.e., condensed matter physics systems whose low-energy excitations have a gapless linear, relativisticlike linear dispersion relation and where electrons are confined to a plane $\left(d_{e}=2+1\right)$ while interacting via the exchange of photons that can travel through a $d_{\gamma}=(3+1)$-dimensional bulk. A prototypical example includes graphene [23-25]. Nowadays, planar Dirac liquids are well-observed experimentally and are under active study in, e.g., (artificial) graphenelike materials [26], surface states of topological insulators [27], and half-filled fractional quantum Hall systems [28]. 
Interest in $\mathrm{RQED}_{4,3}$ also comes from its connections to $\mathrm{QED}_{3}$ [29], which is quite often used as an effective field theory of high temperature superconductors [30-32].

More specifically, we will focus on the case of massless $\mathrm{RQED}_{4,3}$. Within the condensed matter context, a vanishing fermion mass implies long-ranged (unscreened) interactions among the electrons in the absence of doping (the so-called intrinsic case). These interactions in turn enforce the flow of the Fermi velocity, e.g., $v \approx c / 300$ at experimentally accessible scales for graphene, to the velocity of light, $c$, deep in the infrared (IR) with a corresponding flow of the fine structure constant, e.g., $\alpha_{g} \approx$ $e^{2} / 4 \pi \epsilon \hbar v \approx 2.2$ for graphene, to the usual fine structure constant, $\alpha_{\mathrm{em}} \approx 1 / 137$. Within this context, it is this IR Lorentz invariant fixed point [33] that can be described by massless $\mathrm{RQED}_{4,3}$ [22]. A thorough understanding of this fixed point is a prerequisite to set on a firm ground the study of the physics away from the fixed point which is closer to the experimental reality. But this is more difficult to study theoretically; see, e.g., [34], the interesting new work [35], and also the recent reviews in Refs. [36,37].

The gauge-covariance of the fermion propagator of massless reduced QED has already been considered in [12]. Here, we carefully reconsider this problem using the LKF transformation in the framework of dimensional regularization. We not only focus on the bare propagator but also on the renormalized one and provide a detailed comparison between the weak-coupling expansion of LKFtransformed quantities and earlier exact perturbative calculations [21,22].

This paper is organized as follows. In Sec. II, we start by introducing the position space LKF transformation for the general case of reduced QED theories and then derive its momentum space representation for $\mathrm{QED}_{4,3}$ that will be the main subject of focus from there on. In Sec. III, a weakcoupling expansion of this transformation is performed up to two loops in the $\overline{\mathrm{MS}}$ scheme and its matching with existing perturbative results are discussed. A similar task is carried out in Sec. IV for the renormalization constant and the renormalized propagator. Additionally, we present a proof of the purely one-loop gauge dependence of the fermion anomalous dimension in reduced QED. Finally, in Sec. V, we summarize our results and conclude. For completeness, various other choices of scales are presented in Appendix A and in Appendix B the LKF transformation for reduced scalar QED is derived.

\section{LKF TRANSFORMATION FOR REDUCED QED}

We have the following action for reduced $\mathrm{QED}_{d_{r}, d_{\mathrm{e}}}$ :

$$
\begin{aligned}
S_{\mathrm{RQED}}= & \int d^{d_{\mathrm{e}}} x \bar{\psi}_{\sigma} \mathrm{i} D_{\mu} \gamma^{\mu} \psi^{\sigma} \\
& +\int d^{d_{\gamma}} x\left[-\frac{1}{4} F_{\mu \nu} F^{\mu \nu}-\frac{1}{2 \xi}\left(\partial_{\mu} A^{\mu}\right)^{2}\right],
\end{aligned}
$$

where $\xi$ is the gauge fixing parameter and the flavor index $\sigma$ runs from 1 to $N_{F}$. In Eq. (1), the volume elements show that the fermion fields $\psi_{\sigma}$ are confined to $d_{\mathrm{e}}$ dimensions whereas the gauge field mediates the interaction through $d_{\gamma}$ space-time dimensions. In explicit form, the (Euclidean space) photon propagator in reduced QEDs reads [20], [38]

$$
\tilde{D}_{0}^{\mu \nu}(q)=\frac{1}{(4 \pi)^{\varepsilon_{e}}} \frac{\Gamma\left(1-\varepsilon_{e}\right)}{\left(q^{2}\right)^{1-\varepsilon_{e}}}\left(\delta^{\mu \nu}-(1-\tilde{\xi}) \frac{q^{\mu} q^{\nu}}{q^{2}}\right),
$$

where $\tilde{\xi}=\varepsilon_{e}+\left(1-\varepsilon_{e}\right) \xi$ is the reduced gauge fixing parameter while we may refer to the original gauge fixing parameter $\xi$ as the bulk one. In the following, all results will be presented in Euclidean space $\left(d_{\gamma}=4-2 \varepsilon_{\gamma}, d_{\mathrm{e}}=\right.$ $d_{\gamma}-2 \varepsilon_{\mathrm{e}}$ ) for $\mathrm{QED}_{d_{\gamma}, d_{\mathrm{e}}}$ by analogy with the case of $\mathrm{QED}_{4}$.

\section{A. LKF transformation in position space}

We assume that the fermion propagator $S_{F}(p, \xi)$ in some gauge $\xi$ takes the following general form:

$$
S_{F}(p, \xi)=-\frac{\mathrm{i}}{\hat{p}} P(p, \xi),
$$

where $\hat{p}=\gamma^{\mu} p_{\mu}$, which contains Dirac $\gamma$ matrices, has been factored out and $P(p, \xi)$ is a scalar function, i.e., its momentum dependence is only via $p^{2}$. By analogy, the position-space representation $S_{F}(x, \xi)$ of the fermion propagator can be written as

$$
S_{F}(x, \xi)=\hat{x} X(x, \xi),
$$

where $S_{F}(x, \xi)$ and $S_{F}(p, \xi)$ are related to each other with the help of the Fourier transform

$$
\begin{aligned}
& S_{F}(p, \xi)=\int \frac{\mathrm{d}^{d} x}{(2 \pi)^{d / 2}} e^{\mathrm{i} p x} S_{F}(x, \xi), \\
& S_{F}(x, \xi)=\int \frac{\mathrm{d}^{d} p}{(2 \pi)^{d / 2}} e^{-\mathrm{i} p x} S_{F}(p, \xi) .
\end{aligned}
$$

In position space, the LKF transformation [1,2] connects in a very simple way the representations of fermion propagators written for different gauge parameters $\xi$ and $\eta$. In dimensional regularization, it takes the following form:

$$
S_{F}(x, \xi)=S_{F}(x, \eta) e^{D(x)-D(0)},
$$

where [12]

$$
D(x)=f\left(\varepsilon_{\mathrm{e}}\right) \Delta e^{2} \mu^{4-d_{\gamma}} \int \frac{\mathrm{d}^{d_{\mathrm{e}}} p}{(2 \pi)^{d_{\mathrm{e}}}} \frac{e^{-\mathrm{i} p x}}{\left(p^{2}\right)^{2-\varepsilon_{\mathrm{e}}}}, \quad \Delta=\xi-\eta,
$$


and the prefactor is given by

$$
f\left(\varepsilon_{\mathrm{e}}\right)=\frac{\Gamma\left(2-\varepsilon_{\mathrm{e}}\right)}{(4 \pi)^{\varepsilon_{\mathrm{e}}}}
$$

and follows from the longitudinal part of the photon propagator in Eq. (2) above. As in the case of $\mathrm{QED}_{4}$, see [11], $D(0)$ is proportional to the massless tadpole and therefore vanishes in dimensional regularization. Hence, Eq. (6) takes the simpler form

$$
S_{F}(x, \xi)=S_{F}(x, \eta) e^{D(x)},
$$

and the remaining task is to compute $D(x)$. This can be achieved using the following simple formulas for the Fourier transform of massless propagators (see, for example, Ref. [39]):

$$
\begin{gathered}
\int \mathrm{d}^{d} x \frac{e^{\mathrm{i} p x}}{x^{2 \alpha}}=\frac{2^{2 \tilde{\alpha}} \pi^{d / 2} a(\alpha)}{p^{2 \tilde{\alpha}}}, \quad a(\alpha)=\frac{\Gamma(\tilde{\alpha})}{\Gamma(\alpha)}, \quad \tilde{\alpha}=\frac{d}{2}-\alpha, \\
\int \mathrm{d}^{d} p \frac{e^{-\mathrm{i} p x}}{p^{2 \alpha}}=\frac{2^{2 \tilde{\alpha}} \pi^{d / 2} a(\alpha)}{x^{2 \tilde{\alpha}}} .
\end{gathered}
$$

We would like to note that the use of the Euclidean metric simplifies the Fourier transforms, thereby illuminating the appearance of additional factors such as $\mathrm{i}^{k}$, where $\mathrm{i}$ is the imaginary unit and the factor $k$ is $\varepsilon$ independent.

So, for RQED ${ }_{d_{y}, d_{\mathrm{e}}}$, we have

$$
\begin{aligned}
D(x) & =\Delta e^{2}\left(\mu^{2} x^{2}\right)^{2-d_{\gamma} / 2} \frac{\Gamma\left(d_{\gamma} / 2-2\right)}{2^{4}(\pi)^{d_{\gamma} / 2}} \\
& =\Delta A \Gamma\left(d_{\gamma} / 2-2\right)\left(\pi \mu^{2} x^{2}\right)^{2-d_{\gamma} / 2},
\end{aligned}
$$

where $A=\frac{\alpha_{\mathrm{em}}}{4 \pi}=\frac{e^{2}}{(4 \pi)^{2}}$.

Making the dependence on the parameter $\varepsilon$ explicit (here and below we shall set $\varepsilon_{\gamma} \equiv \varepsilon$ and $d_{\gamma} \equiv d$ ), we finally arrive at the expression

$$
D(x)=-\frac{\Delta A}{\varepsilon} \Gamma(1-\varepsilon)\left(\pi \mu^{2} x^{2}\right)^{\varepsilon} .
$$

Remarkably, the parameter $\varepsilon_{\mathrm{e}}$ has completely disappeared and Eq. (12) has exactly the same form as in $\mathrm{QED}_{4}$ with a common factor $\Delta A$, accompanied by the singularity $\varepsilon^{-1}$, contributing to $D(x)$.

Hereafter, we shall only consider the case $d_{\mathrm{e}}=3$, i.e., $\mathrm{RQED}_{d_{\gamma}, 3}$, which corresponds (as $d_{\gamma} \rightarrow 4$ ) to the ultrarelativistic limit of graphene (see Ref. [20]). Note that, as it was shown in [34], an application of dimensional regularization is very convenient in the nonrelativistic limit as well, i.e., where the particles interact via the (instantaneous) Coulomb interaction.

\section{B. LKF transformation in momentum space}

Let $S_{F}(p, \eta)$, the fermion propagator for some gauge parameter $\eta$ and external momentum $p$, take the form (3) with $P(p, \eta)$ having an expansion

$$
P(p, \eta)=\sum_{m=0}^{\infty} a_{m}(\eta) A^{m}\left(\frac{\tilde{\mu}^{2}}{p^{2}}\right)^{m \varepsilon},
$$

which is appropriate for the massless case relevant to the present study (as explained in the Introduction, it corresponds to the ultrarelativistic limit of planar Dirac liquids) [40]. In Eq. (13), the $a_{m}(\eta)$ are coefficients of the loop expansion and $\tilde{\mu}$ the renormalization scale,

$$
\tilde{\mu}^{2}=4 \pi \mu^{2},
$$

which lies somewhere between the MS scale $\mu$ and $\overline{\mathrm{MS}}$ scale $\bar{\mu}$. Then, the LKF transformation shows that for another gauge parameter $\xi$, the result has the form

$$
P(p, \xi)=\sum_{m=0}^{\infty} a_{m}(\xi) A^{m}\left(\frac{\tilde{\mu}^{2}}{p^{2}}\right)^{m \varepsilon},
$$

where now

$$
\begin{aligned}
a_{m}(\xi)= & a_{m}(\eta) \frac{\Gamma(3 / 2-(m+1) \varepsilon)}{\Gamma(1+m \varepsilon)} \\
& \times \sum_{l=0}^{\infty} \frac{\Gamma(1+(m+l) \varepsilon) \Gamma^{l}(1-\varepsilon)}{l ! \Gamma(3 / 2-(m+l+1) \varepsilon)} \frac{(\Delta A)^{l}}{(-\varepsilon)^{l}}\left(\frac{\tilde{\mu}^{2}}{p^{2}}\right)^{l \varepsilon} .
\end{aligned}
$$

In order to derive Eq. (16), we used the fermion propagator $S_{F}(p, \eta)$ with $P(p, \eta)$ given by Eq. (13) took the inverse Fourier transform to $S_{F}(x, \eta)$ and applied the LKF transformation (9) in position space. As a final step, we took the Fourier transform back to momentum space and obtained $S_{F}(p, \xi)$ with $P(p, \xi)$ in (15). Let us also note that expansions similar to (13) and (16) can also be expressed in Minkowski space with the help of the replacement $p^{2} \rightarrow-p^{2}$.

\section{C. $\overline{\mathrm{MS}}$ scheme}

Now let us focus on the $\overline{\mathrm{MS}}$ scale $\bar{\mu}$, which is equal (in the most standard definition) to

$$
\bar{\mu}^{2}=\tilde{\mu}^{2} e^{-\gamma_{E}},
$$

where $\gamma_{E}$ is the Euler-Mascheroni constant. As is well known, the $\overline{\mathrm{MS}}$ scale completely subtracts out the universal factors of $\gamma_{E}$ from the $\varepsilon$-expansions.

In the $\overline{\mathrm{MS}}$ scheme, we can rewrite the result (16) in the following form: 


$$
\begin{aligned}
a_{m}(\xi)= & a_{m}(\eta) \sum_{l=0}^{\infty} \frac{(1-2(m+1) \varepsilon)}{(1-2(m+l+1) \varepsilon)} \\
& \times \tilde{\Phi}(m, l, \varepsilon) \frac{(\Delta A)^{l}}{(-\varepsilon)^{l} l !}\left(\frac{\bar{\mu}^{2}}{p^{2}}\right)^{l \varepsilon}
\end{aligned}
$$

where we have purposefully extracted the factor $(1-$ $2(m+1) \varepsilon) /(1-2(m+l+1) \varepsilon)$ from $\tilde{\Phi}(m, l, \varepsilon)$ in order to have equal transcendental level, i.e., the same $s$ values of $\zeta_{s}$ in the $\varepsilon$-expansion of $\tilde{\Phi}(m, l, \varepsilon)$ (see below). As will be shown below, the factor $\tilde{\Phi}(m, l, \varepsilon)$ reading

$$
\tilde{\Phi}(m, l, \varepsilon)=\frac{\Gamma(1 / 2-(m+1) \varepsilon) \Gamma(1+(m+l) \varepsilon) \Gamma^{l}(1-\varepsilon)}{\Gamma(1+m \varepsilon) \Gamma(1 / 2-(m+l+1) \varepsilon) e^{-l \gamma_{E} \varepsilon}}
$$

can be written as an expansion in the $\zeta_{i}(i \geq 2)$ Euler constants. Note that the $\gamma_{E}$-dependent term arises from the redefinition (17) of the scale $\tilde{\mu} \rightarrow \bar{\mu}$.

At this point, it is convenient to reexpress the $\Gamma$ functions with arguments close to half-integer ones using the standard property (Legendre duplication formula)

$$
\Gamma(2 \alpha)=\frac{2^{2 \alpha-1}}{\Gamma(1 / 2)} \Gamma(\alpha) \Gamma(\alpha+1 / 2),
$$

which leads to the following relation:

$$
\begin{aligned}
\frac{\Gamma(1 / 2-(m+1) \varepsilon)}{\Gamma(1 / 2-(m+l+1) \varepsilon)}= & \frac{1}{2^{2 l \varepsilon}} \frac{\Gamma(1-2(m+1) \varepsilon)}{\Gamma(1-(m+1) \varepsilon)} \\
& \times \frac{\Gamma(1-(m+l+1) \varepsilon)}{\Gamma(1-2(m+l+1) \varepsilon)} .
\end{aligned}
$$

Then, we may write

$$
\tilde{\Phi}(m, l, \varepsilon)=\frac{1}{4^{l \varepsilon}} \Phi(m, l, \varepsilon),
$$

with

$$
\begin{aligned}
\Phi(m, l, \varepsilon)= & \frac{\Gamma(1-2(m+1) \varepsilon) \Gamma(1+(m+l) \varepsilon)}{\Gamma(1-(m+1) \varepsilon) \Gamma(1+m \varepsilon)} \\
& \times \frac{\Gamma(1-(m+l+1) \varepsilon) \Gamma^{l}(1-\varepsilon)}{\Gamma(1-2(m+l+1) \varepsilon) e^{-l \gamma_{E} \varepsilon}}
\end{aligned}
$$

and Eq. (18) can be represented as

$$
a_{m}(\xi)=a_{m}(\eta) \sum_{l=0}^{\infty} \bar{\Phi}(m, l, \varepsilon) \frac{(\Delta A)^{l}}{(-\varepsilon)^{l} l !}\left(\frac{\bar{\mu}^{2}}{4 p^{2}}\right)^{l \varepsilon},
$$

with

$$
\bar{\Phi}(m, l, \varepsilon)=\frac{1-2(m+1) \varepsilon}{1-2(m+l+1) \varepsilon} \Phi(m, l, \varepsilon) .
$$

We would like to draw the attention of the reader to the redefinition of the argument in the r.h.s. of (24): $\bar{\mu}^{2} / p^{2} \rightarrow \bar{\mu}^{2} /\left(4 p^{2}\right)$. Such a redefinition amounts to subtracting factors of $\ln 2$. As we shall see below, it agrees with the exact perturbative calculations done with the $\overline{\mathrm{MS}}$ scale (see [22]). Note that the latter include an additional negative sign for momentum squared in the denominator because the results of [22] were given in Minkowski space. Therefore, $p_{E}^{2}=-p_{M}^{2}$ under Wick rotations in the mostly minus signature that was used in that paper.

\section{D. $\varepsilon$-expansion}

Let us recall that the $\Gamma$-function $\Gamma(1+\beta \varepsilon)$ has the following expansion around 1 :

$\Gamma(1+\beta \varepsilon)=\exp \left[-\gamma_{E} \beta \varepsilon+\sum_{s=2}^{\infty}(-1)^{s} \eta_{s} \beta^{s} \varepsilon^{s}\right], \quad \eta_{s}=\frac{\zeta_{s}}{s}$.

So, the factor $\Phi(m, l, \varepsilon)$ can be written as

$$
\Phi(m, l, \varepsilon)=\exp \left[\sum_{s=2}^{\infty} \eta_{s} p_{s}(m, l) \varepsilon^{s}\right]
$$

where (but now including $s \geq 1$ )

$$
\begin{aligned}
p_{s}(m, l)= & \left(2^{s}-1\right)\left\{(m+1)^{s}-(m+l+1)^{s}\right\} \\
& +\left(1+\delta_{s}^{1}\right) l+(-1)^{s}\left\{(m+l)^{s}-m^{s}\right\}
\end{aligned}
$$

( $\delta_{s}^{1}$ is the Kronecker symbol) and indeed,

$$
p_{1}(m, l)=0, \quad p_{2}(m, l)=-l(4 m+5+2 l),
$$

i.e., the $\overline{\mathrm{MS}}$ scale takes out the Euler constant $\gamma_{E}$ from consideration.

As can be seen from Eq. (27), the factor $\Phi(m, l, \varepsilon)$ contains $\zeta_{s}$ values with the same weight $s$ in front of $\varepsilon^{s}$. This is rather similar to what was found in Ref. [41]. In some cases, such a property allows to derive results without any detailed calculations (as in Ref. [42]). In other cases, it simplifies the structure of the results, which can be predicted as an ansatz in a very simple way (see Refs. $[43,44])$. For recent applications of this property to QCD and super Yang-Mills, see the papers [45] and references and discussions therein.

Recently, this property was also applied to the LKF transformation of $\mathrm{QED}_{4}$ in [11] by some of the present authors. Combined with an appropriate choice of scale, it led to an all-order proof [11] that the perturbative series can be exactly expressed in terms of a hatted transcendental 
basis that eliminates all even $\zeta$ values, i.e., the no- $\pi$ theorem [15-19]. In the case of $\mathrm{QED}_{4,3}$, the situation is not so simple. As can be seen from Eq. (29), the fact that $p_{2}(m, l) \neq 0$ means that $\zeta_{2}$ values cannot be subtracted out, unlike in the even-dimensional case [11]. As shown in Appendix A, other choices of scale are possible but do not further simplify the transcendental structure of this (partially) odd dimensional theory; see also Ref. [46] for an early study.

Thus, in this section, we have obtained a series representation, Eq. (24), for the LKF transformation of the fermion propagator of reduced $\mathrm{QED}_{4,3}$ in the $\overline{\mathrm{MS}}$ scheme (see Appendix A for other choices of scales and Appendix B for an analogous expression in the case of reduced scalar QED). We now need to verify that the gauge dependence produced by this transformation agrees with exact perturbative results (known in the literature up to the two-loop order). The next two sections are devoted to this task.

\section{LKF TRANSFORMATION FOR THE BARE FERMION PROPAGATOR}

\section{A. Bare fermion propagator}

The calculations of the photon and fermion propagators in the framework of the reduced QED have been done in Refs. [20-22], respectively (see also the recent reviews in $[36,37])$.

The fermion propagator (3) can be represented in the following form:

$$
P(p, \xi)=\frac{1}{1-\Sigma_{V}\left(p^{2}, \xi\right)},
$$

where the fermion self-energy $\Sigma_{V}\left(p^{2}, \xi\right)$ can be written with next-to-leading order (NLO) accuracy as

$$
\Sigma_{V}\left(p^{2}, \xi\right)=\Sigma_{1 V}\left(p^{2}, \xi\right)+\Sigma_{2 V}\left(p^{2}, \xi\right) .
$$

Here, $\Sigma_{1 V}\left(p^{2}, \xi\right)$ and $\Sigma_{2 V}\left(p^{2}, \xi\right)$ are the one- and two-loop contributions to the self-energy. Their bare contributions can be represented in the following simple form [47]:

$$
\Sigma_{l V}\left(p^{2}, \xi\right)=A^{l} \bar{\Sigma}_{l V}(\xi)\left(\frac{\bar{\mu}^{2}}{4 p^{2}}\right)^{l \varepsilon}
$$

where the coefficients $\bar{\Sigma}_{l V}(\xi)$ are now just expressions without $\bar{\mu}$ or $p$ dependence anymore.

The one-loop term $\bar{\Sigma}_{1 V}(\xi)$ takes the following form [22]:

$$
\begin{aligned}
\bar{\Sigma}_{1 V}(\xi)= & \frac{1-3 \xi}{3 \varepsilon}+\frac{10}{9}-2 \xi \\
& +\left(\frac{112}{27}-8 \xi-\frac{7(1-3 \xi)}{6} \zeta_{2}\right) \varepsilon+O\left(\varepsilon^{2}\right) .
\end{aligned}
$$

The two-loop term, $\bar{\Sigma}_{2 V}(\xi)$, can be represented as a sum of three contributions corresponding to three distinct Feynman diagrams [22],

$$
\bar{\Sigma}_{2 V}(\xi)=\bar{\Sigma}_{2 a V}(\xi)+\bar{\Sigma}_{2 b V}(\xi)+\bar{\Sigma}_{2 c V}(\xi),
$$

with

$$
\begin{aligned}
\bar{\Sigma}_{2 a V}(\xi)= & -4 N_{F} \zeta_{2}\left(\frac{1}{\varepsilon}+2 \ln 4\right)+O(\varepsilon), \\
\bar{\Sigma}_{2 b V}(\xi)= & \frac{(1-3 \xi)^{2}}{18 \varepsilon^{2}}+\left(\frac{11}{27}-\frac{7 \xi}{9}\right) \frac{1-3 \xi}{\varepsilon}+\frac{206}{81} \\
& +2(7 \xi-6) \xi-\frac{(1-3 \xi)^{2}}{2} \zeta_{2}+O(\varepsilon), \\
\bar{\Sigma}_{2 c V}(\xi)= & -\frac{(1-3 \xi)^{2}}{9 \varepsilon^{2}}+\left(-\frac{37}{27}+\frac{(34-39 \xi) \xi}{9}\right) \frac{1}{\varepsilon} \\
& -\frac{1390}{81}+\frac{532 \xi}{27}-22 \xi^{2}+\frac{71+21 \xi(3 \xi-2)}{9} \zeta_{2} \\
& +O(\varepsilon) .
\end{aligned}
$$

We note that the part $\bar{\Sigma}_{2 a V}(\xi)$ is $\xi$ independent and, thus, the full result can be represented in the form

$$
\bar{\Sigma}_{2 V}(\xi)=\bar{\Sigma}_{2 a V}+\bar{\Sigma}_{2 b c V}(\xi),
$$

where the contribution

$$
\bar{\Sigma}_{2 b c V}(\xi)=\bar{\Sigma}_{2 b V}(\xi)+\bar{\Sigma}_{2 c V}(\xi)
$$

has the following expression:

$$
\begin{aligned}
\bar{\Sigma}_{2 b c V}(\xi)= & -\frac{(1-3 \xi)^{2}}{18 \varepsilon^{2}}-\frac{2}{27 \varepsilon}(13-3 \xi(8-9 \xi)) \\
& -\frac{8}{81}(148-3 \xi(26-27 \xi)) \\
& +\frac{\zeta_{2}}{18}\left(128+5(1-3 \xi)^{2}\right)+O(\varepsilon) .
\end{aligned}
$$

As for the propagator itself, at the NLO approximation, Eq. (30) can be rewritten as

$P(p, \xi)=1+\Sigma_{1 V}\left(p^{2}, \xi\right)+\Sigma_{1 V}^{2}\left(p^{2}, \xi\right)+\Sigma_{2 V}\left(p^{2}, \xi\right)+\cdots$,

where $\Sigma_{1 V}\left(p^{2}\right)$ has the form (32) with $l=1$ and the contribution $\Sigma_{1 V}^{2}\left(p^{2}\right)+\Sigma_{2 V}\left(p^{2}\right)$ can be represented as

$\Sigma_{1 V}^{2}\left(p^{2}, \xi\right)+\Sigma_{2 V}\left(p^{2}, \xi\right)=A^{2}\left(\bar{\Sigma}_{2 a V}+\widetilde{\Sigma}_{2 b c V}(\xi)\right)\left(\frac{\mu^{2}}{4 p^{2}}\right)^{2 \varepsilon}$ 
with

$$
\begin{aligned}
\widetilde{\Sigma}_{2 b c V}(\xi)= & \bar{\Sigma}_{2 b c V}(\xi)+\bar{\Sigma}_{1 V}^{2}(\xi) \\
= & \frac{(1-3 \xi)^{2}}{18 \varepsilon^{2}}-\frac{1}{\varepsilon}\left(\frac{2}{9}+\frac{16}{9} \xi-2 \xi^{2}\right) \\
& +\zeta_{2}\left(\frac{64}{9}-\frac{1}{2}(1-3 \xi)^{2}\right) \\
& -\frac{4}{81}(215+3 \xi(70-81 \xi))+O(\varepsilon) .
\end{aligned}
$$

Let us note that the last term $\widetilde{\Sigma}_{2 b c V}(\xi)$ contains all the $\xi$ dependence at the NLO level of accuracy.

\section{B. LKF transformation}

With the help of the results of Secs. II C and III A above, we can deduce that the one- and two-loop results for the fermion propagator in two different gauges are related to each other in the following way:

$$
\begin{aligned}
\bar{\Sigma}_{1 V}(\xi) & =\bar{\Sigma}_{1 V}(\eta)+\bar{\Sigma}_{0 V}(\eta) \bar{\Phi}(0,1, \varepsilon) \frac{\Delta}{(-\varepsilon)}, \\
\bar{\Sigma}_{2 V}(\xi)+\bar{\Sigma}_{1 V}^{2}(\xi)= & \bar{\Sigma}_{2 V}(\eta)+\bar{\Sigma}_{1 V}^{2}(\eta)+\bar{\Sigma}_{1 V}(\eta) \bar{\Phi}(1,1, \varepsilon) \\
& \times \frac{\Delta}{(-\varepsilon)}+\bar{\Sigma}_{0 V}(\eta) \bar{\Phi}(0,2, \varepsilon) \frac{\Delta^{2}}{2(-\varepsilon)^{2}},
\end{aligned}
$$

with $\bar{\Sigma}_{0 V}(\eta)=1$.

Taking $\eta=0$, i.e., starting from the Landau gauge and the fact that the contribution $\bar{\Sigma}_{2 a V}$ is gauge invariant, we have that

$$
\begin{aligned}
\bar{\Sigma}_{1 V}(\xi)= & \bar{\Sigma}_{1 V}(\xi=0)+\bar{\Phi}(0,1, \varepsilon) \frac{\xi}{(-\varepsilon)} \\
\widetilde{\Sigma}_{2 b c V}(\xi)= & \widetilde{\Sigma}_{2 b c V}(\xi=0)+\bar{\Sigma}_{1 V}(\xi=0) \bar{\Phi}(1,1, \varepsilon) \frac{\xi}{(-\varepsilon)} \\
& +\bar{\Phi}(0,2, \varepsilon) \frac{\xi^{2}}{2(-\varepsilon)^{2}},
\end{aligned}
$$

where the results for $\bar{\Sigma}_{1 V}(\xi=0)$ and $\widetilde{\Sigma}_{2 b c V}(\xi=0)$ can be obtained from Eqs. (33) and (41) after setting $\xi=0$. This yields

$$
\begin{gathered}
\bar{\Sigma}_{1 V}(\xi=0)=\frac{1}{3 \varepsilon}+\frac{10}{9}+\left(\frac{112}{27}-\frac{7}{6} \zeta_{2}\right) \varepsilon+O\left(\varepsilon^{2}\right), \\
\widetilde{\Sigma}_{2 b c V}(\xi=0)=\frac{1}{18 \varepsilon^{2}}-\frac{2}{9 \varepsilon}+\frac{119}{18} \zeta_{2}-\frac{860}{81}+O(\varepsilon) .
\end{gathered}
$$

With the help of Eqs. (25), (27), and (28), we find that the expansions of $\bar{\Phi}(m, l, \varepsilon)$ for the cases of interest read

$$
\begin{aligned}
& \bar{\Phi}(0,1, \varepsilon)=1+2 \varepsilon+\left(8-\frac{7}{2} \zeta_{2}\right) \varepsilon^{2} \\
& \bar{\Phi}(1,1, \varepsilon)=1+2 \varepsilon+\left(12-\frac{11}{2} \zeta_{2}\right) \varepsilon^{2} \\
& \bar{\Phi}(0,2, \varepsilon)=1+4 \varepsilon+\left(24-9 \zeta_{2}\right) \varepsilon^{2}
\end{aligned}
$$

Then Eq. (43), together with the expressions of $\bar{\Sigma}_{1 V}(\xi=0)$ and $\widetilde{\Sigma}_{2 b c V}(\xi=0)$ in (44a) and $(44 \mathrm{~b})$ as well as the $\varepsilon$-expansions of Eq. (45), immediately allows to reproduce the full results for $\bar{\Sigma}_{1 V}(\xi)$ and $\widetilde{\Sigma}_{2 b c V}(\xi)$ presented in the previous sections, Eqs. (33) and (41).

Thus, we have verified that the bare results for $\bar{\Sigma}_{1 V}(\xi)$ and $\widetilde{\Sigma}_{2 b c V}(\xi)$ are exactly in agreement with the LKF transformation (using dimensional regularization, our derivations proceed without any replacements involving a cutoff parameter $\Lambda$ and the scale $\mu$ as in the case of Ref. [12]).

\section{LKF TRANSFORMATION AND RENORMALIZATION}

\section{A. Renormalized fermion propagator in momentum space}

Since all renormalized results are constructed from the bare ones through the Bogoliubov-Parasiuk-HeppZimmermann (BPHZ) procedure (for a definition of the procedure, see, for example, Refs. [22,37]), all results including the renormalized ones must be in agreement with the LKF transformation too.

In order to show this explicitly to the two-loop order, let us first note that the fermion propagator given by Eq. (3) is the unrenormalized one. It can be conveniently factored as

$$
P(p, \xi)=Z_{\psi}(A, \xi) P_{r}(p, \xi),
$$

where we have taken into account the fact that $A$ and $\xi$ are not renormalized in $\mathrm{QED}_{4,3}$, i.e., $A_{r} \equiv A$ and $\xi_{r} \equiv \xi$. In Eq. (46), the renormalization constant $Z_{\psi}(A, \xi)$ and the renormalized fermion propagator $P_{r}(p, \xi)$ can be expanded as

$Z_{\psi}(A, \xi)=1+\sum_{l=1}^{+\infty} Z_{l \psi}(\xi) A^{l} ; \quad Z_{l \psi}(\xi)=\sum_{j=-l}^{-1} Z_{\psi}^{(l, j)}(\xi) \varepsilon^{j}$,

$P_{r}(p, \xi)=1+\sum_{l=1}^{+\infty} P_{l r}(p, \xi) A^{l}$

$P_{l r}(p, \xi)=\sum_{j=0}^{+\infty} P_{r}^{(l, j)}(p, \xi) \varepsilon^{j}$.

The renormalization constant and renormalized propagator have been computed [22] up to two loops in reduced QED, 
for arbitrary $\varepsilon$ at one loop and to $\mathrm{O}\left(\varepsilon^{0}\right)$ for the propagator. The one-loop expressions read [22]

$$
\begin{aligned}
Z_{1 \psi}(\xi)= & \frac{1-3 \xi}{3 \varepsilon} \\
P_{1 r}(p, \xi)= & \frac{10}{9}-\frac{1-3 \xi}{3} L_{p}-2 \xi \\
& +\left(\frac{112}{27}-\frac{7 \zeta_{2}}{6}-\frac{10 L_{p}}{9}+\frac{L_{p}^{2}}{6}\right. \\
& \left.-\xi\left(8-\frac{7 \zeta_{2}}{2}-2 L_{p}+\frac{L_{p}^{2}}{2}\right)\right) \varepsilon+\mathrm{O}\left(\varepsilon^{2}\right)
\end{aligned}
$$

where $L_{p}=\ln \left(4 p^{2} / \bar{\mu}^{2}\right)$. The two-loop expressions are given by [22]

$$
\begin{aligned}
Z_{2 \psi}(\xi)= & \frac{(1-3 \xi)^{2}}{18 \varepsilon^{2}}-\frac{4}{\varepsilon}\left(N_{F} \zeta_{2}+\frac{4}{27}\right) \\
P_{2 r}(p, \xi)= & 8 N_{F} \zeta_{2}\left(L_{p}-2 \ln 2\right)-12+7 \zeta_{2}+\frac{22}{27} L_{p} \\
& +\frac{L_{p}^{2}}{18}-\frac{\xi}{9}\left(32-6 \zeta_{2}-16 L_{p}+3 L_{p}^{2}\right) \\
& +\xi^{2}\left(4-\zeta_{2}-2 L_{p}+\frac{L_{p}^{2}}{2}\right)+\mathrm{O}(\varepsilon) .
\end{aligned}
$$

Let us further note that these expressions allow one to compute the fermion anomalous dimension up to two loops with the help of the relation,

$\gamma_{\psi}(A, \xi)=\sum_{l=1}^{\infty} \gamma_{\psi, l}(\xi) A^{l}, \quad \gamma_{\psi, l}(\xi)=2 l Z_{\psi}^{(l,-1)}(\xi)$,

yielding [22]

$\gamma_{\psi}(A, \xi)=2 A \frac{1-3 \xi}{3}-16 A^{2}\left(N_{F} \zeta_{2}+\frac{4}{27}\right)+\mathrm{O}\left(A^{3}\right)$.

\section{B. LKF transformation in momentum space}

We shall now determine the LKF transformations of $Z_{l \psi}(\xi)$ and $P_{l r}(p, \xi)$ up to two loops and compare the obtained results with those of the last subsection. In order to proceed, we first note that, at NLO, Eq. (46) can be written as

$$
\begin{aligned}
P(p, \xi)= & 1+A\left(Z_{1 \psi}(\xi)+P_{1 r}(p, \xi)\right) \\
& +A^{2}\left(Z_{2 \psi}(\xi)+Z_{1 \psi}(\xi) P_{1 r}(p, \xi)\right. \\
& \left.+P_{2 r}(p, \xi)\right)+\mathrm{O}\left(A^{3}\right) .
\end{aligned}
$$

Comparing (52) with (39) and using the notations of (32) then yields

$$
\begin{aligned}
Z_{1 \psi}(\xi)+P_{1 r}(p, \xi) & =\bar{\Sigma}_{1 V}(\xi)\left(\frac{\bar{\mu}^{2}}{4 p^{2}}\right)^{\varepsilon} \\
Z_{2 \psi}(\xi)+Z_{1 \psi}(\xi) P_{1 r}(p, \xi)+P_{2 r}(p, \xi) & =\widetilde{\Sigma}_{2 V}(\xi)\left(\frac{\bar{\mu}^{2}}{4 p^{2}}\right)^{2 \varepsilon}, \\
\widetilde{\Sigma}_{2 V} & =\bar{\Sigma}_{2 V}+\bar{\Sigma}_{1 V}^{2},
\end{aligned}
$$

where $\bar{\Sigma}_{l V}(\xi)$ has the following $\varepsilon$-expansion:

$$
\bar{\Sigma}_{l V}(\xi)=\sum_{j=-l}^{+\infty} \bar{\Sigma}_{V}^{(l, j)}(\xi) \varepsilon^{j} .
$$

The LKF transformations of $Z_{l \psi}(\xi)$ and $P_{l r}(p, \xi)$ can then be obtained by identifying identical powers of $\varepsilon$ on both sides of Eq. (53).

At one loop, this straightforwardly yields

$$
\begin{aligned}
Z_{1 \psi}(\xi)= & Z_{1 \psi}(0)-\frac{\xi}{\varepsilon}, \\
P_{1 r}(p, \xi)= & P_{1 r}(p, 0)+\left(L_{p}-2\right) \xi \\
& -\xi\left(8-\frac{7 \zeta_{2}}{2}-2 L_{p}+\frac{L_{p}^{2}}{2}\right) \varepsilon+\mathrm{O}\left(\varepsilon^{2}\right),
\end{aligned}
$$

where

$$
\begin{aligned}
Z_{1 \psi}(0)= & \frac{\bar{\Sigma}_{V}^{(1,-1)}(0)}{\varepsilon}, \\
P_{1 r}(p, 0)= & \bar{\Sigma}_{V}^{(1,0)}(0)-L_{p} \bar{\Sigma}_{V}^{(1,-1)}(0) \\
& +\left(\bar{\Sigma}_{V}^{(1,1)}(0)-L_{p} \bar{\Sigma}_{V}^{(1,0)}(0)+\frac{L_{p}^{2}}{2} \bar{\Sigma}_{V}^{(1,-1)}(0)\right) \varepsilon \\
& +\mathrm{O}\left(\varepsilon^{2}\right) .
\end{aligned}
$$

As for the two-loop case, we first note that

$$
\widetilde{\Sigma}_{2 V}(\xi)=\bar{\Sigma}_{2 a V}(\xi)+\widetilde{\Sigma}_{2 b c V}(\xi),
$$

which in component form can be written as

$$
\begin{aligned}
& \widetilde{\Sigma}_{V}^{(2,-2)}(\xi)=\widetilde{\Sigma}_{b c V}^{(2,-2)}(\xi), \\
& \widetilde{\Sigma}_{V}^{(2,-1)}(\xi)=-4 N_{F} \zeta_{2}+\widetilde{\Sigma}_{b c V}^{(2,-1)}(\xi), \\
& \widetilde{\Sigma}_{V}^{(2,0)}(\xi)=-16 N_{F} \zeta_{2} \ln 2+\widetilde{\Sigma}_{b c V}^{(2,0)}(\xi),
\end{aligned}
$$

where we restricted to $\widetilde{\Sigma}_{V}^{(2, j)}$ with $j \leq 0$. Then, using Eq. (43b) yields 


$$
\begin{aligned}
& \widetilde{\Sigma}_{b c V}^{(2,-2)}(\xi)=\widetilde{\Sigma}_{b c V}^{(2,-2)}(0)+\frac{\xi^{2}}{2}-\xi \bar{\Sigma}_{V}^{(1,-1)}(0), \\
& \widetilde{\Sigma}_{b c V}^{(2,-1)}(\xi)=\widetilde{\Sigma}_{b c V}^{(2,-1)}(0)+2 \xi^{2}-\xi \bar{\Sigma}_{V}^{(1,0)}(0)-2 \xi \bar{\Sigma}_{V}^{(1,-1)}(0),
\end{aligned}
$$

$$
\begin{aligned}
\widetilde{\Sigma}_{b c V}^{(2,0)}(\xi)= & \widetilde{\Sigma}_{b c V}^{(2,0)}(0)+\xi^{2}\left(12-\frac{9 \zeta_{2}}{2}\right)-\xi \bar{\Sigma}_{V}^{(1,1)}(0) \\
& -2 \xi \bar{\Sigma}_{V}^{(1,0)}(0)-\xi\left(12-\frac{11 \zeta_{2}}{2}\right) \bar{\Sigma}_{V}^{(1,-1)}(0)
\end{aligned}
$$

We are now in a position to use (53b) and first focus on the renormalization constant. In component form, we obtain

$$
\begin{aligned}
Z_{\psi}^{(2,-2)}(\xi) & =Z_{\psi}^{(2,-2)}(0)-\xi Z_{\psi}^{(1,-1)}(0)+\frac{\xi^{2}}{2}, \\
Z_{\psi}^{(2,-1)}(\xi) & =Z_{\psi}^{(2,-1)}(0)+L_{p}\left(\left(Z_{\psi}^{(1,-1)}(0)\right)^{2}-2 Z_{\psi}^{(2,-2)}(0)\right) \\
& =Z_{\psi}^{(2,-1)}(0),
\end{aligned}
$$

where

$$
\begin{aligned}
& Z_{\psi}^{(2,-2)}(0)=\widetilde{\Sigma}_{b c V}^{(2,-2)}(0) \\
& Z_{\psi}^{(2,-1)}(0)=-4 N_{F} \zeta_{2}+\widetilde{\Sigma}_{b c V}^{(2,-1)}(\xi)-\bar{\Sigma}_{V}^{(1,-1)}(0) \bar{\Sigma}_{V}^{(1,0)}(0) .
\end{aligned}
$$

In Eq. (60b), we used a renormalization constraint arising from the finiteness of the fermion anomalous dimension in the limit $\varepsilon \rightarrow 0$ whereby the coefficients $Z_{\psi}^{(l,-k)}$ for $l>1$ and $k=2, \ldots, l$ may be expressed in terms of coefficients of lower $l$ and $k$. At two loops, there is only one constraint $Z_{\psi}^{(2,-2)}(\xi)=\left(Z_{\psi}^{(1,-1)}(\xi)\right)^{2} / 2$ which, when applied to $(60 \mathrm{~b})$, ensures that the renormalization constant does not depend on $L_{p}$. This agrees with the fact that renormalization constants should not depend on masses and external momenta in the $\overline{\mathrm{MS}}$ scheme [48].

We may proceed in a similar way with the two-loop renormalized fermion propagator. To leading order in the $\varepsilon$-expansion, it has the form

$$
\begin{aligned}
P_{2 r}(p, \xi)= & P_{2 r}(p, 0)+\xi\left(L_{p}-2\right) \bar{\Sigma}_{V}^{(1,0)}(0) \\
& -\xi\left(4-2 \zeta_{2}-2 L_{p}+L_{p}^{2}\right) \bar{\Sigma}_{V}^{(1,-1)}(0) \\
& +\xi^{2}\left(4-\zeta_{2}-2 L_{p}+\frac{L_{p}^{2}}{2}\right)
\end{aligned}
$$

$$
\begin{aligned}
P_{2 r}(p, 0)= & \widetilde{\Sigma}_{V}^{(2,0)}(0)-2 L_{p} \widetilde{\Sigma}_{V}^{(2,-1)}(0)+2 L_{p}^{2} \widetilde{\Sigma}_{V}^{(2,-2)}(0) \\
& -\bar{\Sigma}_{V}^{(1,-1)}(0) \bar{\Sigma}_{V}^{(1,1)}(0) \\
& +L_{p} \bar{\Sigma}_{V}^{(1,-1)}(0) \bar{\Sigma}_{V}^{(1,0)}(0)-\frac{L_{p}^{2}}{2}\left(\bar{\Sigma}_{V}^{(1,-1)}(0)\right)^{2} .
\end{aligned}
$$

We are now in a position to compare the above derived LKF expressions with the exact results presented in Sec. IV A. At one loop, we find a perfect agreement for the terms proportional to $\xi$ between Eqs. (55) and (48). At two loops, we also find a perfect agreement for the terms proportional to $\xi^{2}$ between Eqs. (60) and (49a) on the one hand and between Eqs. (62a) and (49b) on the other hand. These results are in accordance with the fact that at l-loops, the LKF transformation allows to fix exactly all terms proportional to $\xi$.

Moreover, by extracting the values of the coefficients $\bar{\Sigma}_{V}^{(1, j)}(0)$ from Eqs. (44a) and (44b) and substituting them in Eqs. (56), (61), and (62b), we immediately recover from (55), (60), and (62a) the full results of Eqs. (48) and (49).

Finally, we note the remarkable fact that Eq. (60b) does not depend on the gauge fixing parameter. From Eq. (50), this implies that the two-loop fermion anomalous dimension is gauge invariant and is in agreement with (51). Actually, we may extend such a remark to three loops though no exact result is available yet at this order. All calculations done, this yields (in the $\overline{\mathrm{MS}}$ scheme) [49]

$Z_{\psi}^{(3,-3)}(\xi)=\frac{(1-3 \xi)^{3}}{162}$

$Z_{\psi}^{(3,-2)}(\xi)=-\frac{4(1-3 \xi)}{81}\left(27 N_{F} \zeta_{2}+4\right)$,

$Z_{\psi}^{(3,-1)}(\xi)=\bar{\Sigma}_{V}^{(3,-1)}(0)$

$$
+\frac{\zeta_{2}}{9}\left(8 N_{F}(5+6 \ln 2)-\frac{245}{12}\right)+\frac{1076}{243} \text {, }
$$

where the first two terms are easily derived from renormalization constraints [50], while in the third term the Landau gauge coefficient $\bar{\Sigma}_{V}^{(3,-1)}(0)$ is not known at the time of writing. Nevertheless, Eq. (63c) is clearly gauge invariant and so is the three-loop fermion anomalous dimension.

\section{Gauge dependence of $\gamma_{\psi}$}

In the last subsection, the LKF transformation revealed that both the two- and three-loop fermion anomalous dimensions are gauge invariant in reduced QED. We will now show that this gauge invariance extends to all higher 
orders, see Refs. [51,52], for similar proofs in the case of $\mathrm{QED}_{4}$.

We proceed in $x$-space starting from the unrenormalized fermion propagator of Eq. (9). Similar to the $p$-space case, it is conveniently factored as

$$
S_{F}(x, \xi)=Z_{\psi}(A, \xi) S_{F r}(x, \xi) .
$$

Taking the logarithm of Eq. (9) with $D(x)$ given by Eq. (12) and identifying powers of $1 / \varepsilon$ straightforwardly yields

$$
\log Z_{\psi}(A, \xi)=\log Z_{\psi}(A, \eta)-\frac{A \Delta}{\varepsilon},
$$

which simply translates an exponentiation of the gauge dependence at the level of the renormalization constant. At this point, let us recall that

$$
\gamma_{\psi}(A, \xi)=-\beta(A) \frac{\partial \log Z_{\psi}(A, \xi)}{\partial A}-\xi \gamma(A) \frac{\partial \log Z_{\psi}(A, \xi)}{\partial \xi},
$$

where $\beta(A)$ is the beta function and $\gamma(A)$ is the gauge-field anomalous dimension. The latter can be expressed as

$\beta(A)=-2 \varepsilon A+\sum_{l=1}^{\infty} \beta_{l} A^{l+1}, \quad \gamma(A)=\sum_{l=1}^{\infty} \gamma_{l} A^{l}$,

where the coefficients satisfy $\beta_{l}=-\gamma_{l}$ (actually, they even vanish in the case of $\mathrm{RQED}_{4,3}$ ). Substituting Eq. (65) in (66) and using (67) yield

$$
\gamma_{\psi}(A, \xi)=\gamma_{\psi}(A, \eta)-2 A \Delta,
$$

showing that all the gauge dependence is contained in the one-loop contribution while all higher order corrections are indeed gauge invariant.

\section{SUMMARY AND CONCLUSION}

In this paper, we have studied the gauge-covariance of the fermion propagator of reduced QED with the help of the LKF transformation in dimensional regularization. The $x$-space transformation has been derived in the general case of $\mathrm{QED}_{d_{\gamma}, d_{\mathrm{e}}}$ and its structure, Eq. (12), was found to be similar to $\mathrm{QED}_{4}$. Focusing on the odd-dimensional case, $d_{\mathrm{e}}=3$ (together with $d_{\gamma}=4-2 \varepsilon$ ), we have then derived the $p$-space LKF transformation in the form of a series representation for the coefficients of the loop expansion of the propagator in the $\overline{\mathrm{MS}}$ scheme, Eq. (24) (see also Appendix A for other choices of scales and Appendix B for an analogous expression in the case of reduced scalar QED). The series has been expressed in terms of a uniform transcendental factor $\Phi(m, l, \varepsilon)$, Eq. (23). The $\zeta$-structure of the latter [see Eq. (28) and discussion below it] is transcendentally more complicated than in the fourdimensional case [11] as expected from an odddimensional theory [46]. We then performed a two-loop expansion of the transformation for the bare fermion propagator, Eq. (42), and also for the renormalization constant and renormalized propagator, Eqs. (55), (60), and (62). Starting from the Landau gauge $(\xi=0)$ to a general $\xi$-gauge, all these weak-coupling expansions were found to fully agree with previously known exact perturbative results up to the two-loop order. In particular, we have checked that the LKF predicted coefficients of the form $(A \xi)^{l}$ match with the perturbative results. Additionally, we have presented a proof of the purely one-loop gauge dependence of the fermion anomalous dimension in reduced QED, Eq. (68). In conclusion, our analysis and in particular our all order series representations, Eq. (24) and equivalent ones in Appendix A, can of course be used beyond the present two-loop accuracy of perturbative results. They should provide some stringent constraints on future higher order calculations in reduced QED.

\section{ACKNOWLEDGMENTS}

The work of A. J. is supported by the ILP LABEX (under reference ANR-10-LABX-63) through French state funds managed by the ANR within the Investissements d'Avenir programme under reference ANR-11-IDEX-0004-02.

\section{APPENDIX A: OTHER CHOICES OF SCALE}

The calculations in the main text were all performed in the $\overline{\mathrm{MS}}$ scheme on the basis of Eqs. (24) and (25). Following [11], in this appendix we present three other choices of scale which may be more convenient for future higher loop computations: the $g$-scale, the reduced $g$ - (or $\left.g_{R^{-}}\right)$scale and the MV-scale. We therefore define

$$
a_{m}(\xi)=a_{m}(\eta) \sum_{l=0}^{\infty} \bar{\Phi}_{p}(m, l, \varepsilon) \frac{(\Delta A)^{l}}{(-\varepsilon)^{l} l !}\left(\frac{\mu_{p}^{2}}{4 p^{2}}\right)^{l \varepsilon},
$$

with

$$
\bar{\Phi}_{p}(m, l, \varepsilon)=\frac{(1-2(m+1) \varepsilon)}{(1-2(m+l+1) \varepsilon)} \Phi_{p}(m, l, \varepsilon),
$$

where $p=g, g_{R}, \mathrm{MV}$, and the following subsections will focus on the computation of $\Phi_{p}(m, l, \varepsilon)$ for these scales. In the four-dimensional case, these scales are particularly efficient as they allow a complete subtraction of both the Euler constant $\gamma_{E}$ and the $\zeta_{2}$-value [48,53]; see also [11] for a recent application to $\mathrm{QED}_{4}$. As will be shown below, in the present $\left(d_{\gamma}, d_{e}\right)=(4,3)$ case, only the Euler constant is completely subtracted and one cannot avoid the proliferation of $\zeta_{2}$ (as well as $\ln 2$ ) in accordance with the greater transcendental complexity of odd dimensional field theories with respect to even ones [46]. 


\section{1. $g$-scale}

First, let us consider the so-called $G$-scale [53] which subtracts the coefficient in factor of the singularity $1 / \varepsilon$ in the one-loop scalar p-type integral $G(1,1)$. Recalling that

$$
G(\alpha, \beta)=\frac{a(\alpha) a(\beta)}{a(\alpha+\beta-d / 2)},
$$

where $a(\alpha)$ was defined in Eq. (10); the $G$-scale amounts to the following substitution:

$$
\mu_{G}^{2 \varepsilon}=\tilde{\mu}^{2 \varepsilon} \varepsilon G(1,1)=\tilde{\mu}^{2 \varepsilon} \frac{\Gamma^{2}(1-\varepsilon) \Gamma(1+\varepsilon)}{\Gamma(2-2 \varepsilon)} .
$$

Following [15], a slight modification of this scale that was referred to as the $g$-scale in [11] subtracts an additional factor $1 /(1-2 \varepsilon)$ from the one-loop result, i.e.,

$$
\mu_{g}^{2 \varepsilon}=\tilde{\mu}^{2 \varepsilon} \frac{\Gamma^{2}(1-\varepsilon) \Gamma(1+\varepsilon)}{\Gamma(1-2 \varepsilon)} .
$$

With this choice of scale, we have

$$
\Phi_{g}(m, l, \varepsilon)=\Phi(m, l, \varepsilon) \times e^{-l \gamma_{E} \varepsilon} \frac{\Gamma^{l}(1-2 \varepsilon)}{\Gamma^{2 l}(1-\varepsilon) \Gamma^{l}(1+\varepsilon)},
$$

where $\Phi(m, l, \varepsilon)$ is given by Eq. (23). Hence, we obtain

$$
\Phi_{g}(m, l, \varepsilon)=\exp \left[\sum_{s=2}^{\infty} \eta_{s} p_{s}^{(g)}(m, l) \varepsilon^{s}\right],
$$

where (for $s \geq 1$ )

$$
\begin{aligned}
p_{s}^{(g)}(m, l)= & \left(2^{s}-1\right)\left\{l+(m+1)^{s}-(m+l+1)^{s}\right\} \\
& +(-1)^{s}\left\{(m+l)^{s}-m^{s}-l\right\}
\end{aligned}
$$

and

$p_{1}^{(g)}(m, l)=0, p_{s>1}^{(g)}(m, l)=l\left(2^{s}-2-(-1)^{s}\right)+p_{s}(m, l)$,

i.e., the Euler constant $\gamma_{E}$ is completely subtracted as in (28).

\section{Reduced $g$-scale}

For reduced QED, it is more natural to consider the $G$ function $G\left(1,1-\varepsilon_{\mathrm{e}}\right)$ to define a scale, because it corresponds to the one-loop master integral entering the fermion self-energy in this theory. Thus, we write instead

$$
\begin{aligned}
\mu_{g_{R}}^{2 \varepsilon} & =\tilde{\mu}^{2 \varepsilon} \varepsilon(1-2 \varepsilon) G\left(1,1-\varepsilon_{\mathrm{e}}\right) \\
& =\tilde{\mu}^{2 \varepsilon} \frac{\Gamma\left(1-\varepsilon_{\mathrm{e}}-\varepsilon\right) \Gamma(1-\varepsilon) \Gamma(1+\varepsilon)}{\Gamma\left(1-\varepsilon_{\mathrm{e}}-2 \varepsilon\right)},
\end{aligned}
$$

which for $\mathrm{RQED} \mathrm{D}_{4,3}$ becomes

$$
\mu_{g_{R}}^{2 \varepsilon}=\frac{\tilde{\mu}^{2 \varepsilon}}{4^{\varepsilon}} \frac{\Gamma^{2}(1-2 \varepsilon) \Gamma(1+\varepsilon)}{\Gamma(1-4 \varepsilon)} .
$$

This leads to a $\Phi_{g_{R}}$ function,

$\Phi_{g_{R}}(m, l, \varepsilon)=\Phi(m, l, \varepsilon) \times e^{-l \gamma_{E} \varepsilon} 4^{l \varepsilon} \frac{\Gamma^{l}(1-4 \varepsilon)}{\Gamma^{2 l}(1-2 \varepsilon) \Gamma^{l}(1+\varepsilon)}$.

Hence,

$$
\Phi_{g_{R}}(m, l, \varepsilon)=4^{l \varepsilon} \exp \left[\sum_{s=2}^{\infty} p_{s}^{\left(g_{R}\right)}(m, l) \eta_{s} \varepsilon^{s}\right]
$$

where

$$
\begin{aligned}
p_{s}^{\left(g_{R}\right)}(m, l)= & \left(2^{s}-1\right)\left\{(m+1)^{s}-(m+l+1)^{s}\right\} \\
& +\left(2^{s}-1\right)^{2} l+(-1)^{s}\left\{(m+l)^{s}-m^{s}-l\right\},
\end{aligned}
$$

such that

$$
\begin{aligned}
p_{1}^{g_{R}}(m, l) & =0, \\
p_{s>1}^{g_{R}}(m, l) & =l\left(2^{2 s}-2^{s+1}-(-1)^{s}\right)+p_{s}(m, l),
\end{aligned}
$$

i.e., the Euler constant $\gamma_{E}$ is completely subtracted as in (28).

\section{MV-scale}

Yet another convenient choice of scale is the minimal Vladimirov-scale [11] which is defined via the relation

$$
\mu_{\mathrm{MV}}^{2 \varepsilon}=\frac{\tilde{\mu}^{2 \varepsilon}}{\Gamma(1-\varepsilon)} .
$$

With this choice of scale, we have

$$
\Phi_{\mathrm{MV}}(m, l, \varepsilon)=\Phi(m, l, \varepsilon) e^{-l \gamma_{E} \varepsilon} \Gamma^{l}(1-\varepsilon),
$$

where $\Phi(m, l, \varepsilon)$ is given by Eq. (23). Hence,

$$
\Phi_{\mathrm{MV}}(m, l, \varepsilon)=\exp \left[\sum_{s=2}^{\infty} \eta_{s} p_{s}^{(\mathrm{MV})}(m, l) \varepsilon^{s}\right],
$$

where (for $s \geq 1$ ) 


$$
\begin{aligned}
p_{s}^{(\mathrm{MV})}(m, l)= & \left(2^{s}-1\right)\left\{(m+1)^{s}-(m+l+1)^{s}\right\}+2 l \\
& +(-1)^{s}\left\{(m+l)^{s}-m^{s}\right\}
\end{aligned}
$$

and

$p_{1}^{(\mathrm{MV})}(m, l)=0, \quad p_{s>1}^{(\mathrm{MV})}(m, l)=l+p_{s}(m, l)$,

i.e., the Euler constant $\gamma_{E}$ is completely subtracted as in (28).

We can see that in all cases considered, i.e., in $g$, reduced $g$ and MV-scales, we cannot put the values $p_{s=2}^{(\mathrm{i})}(m, l)$ to be zero as it was before in the case of the spinor and scalar QED (see Ref. [11]). Indeed, $p_{s=2}(m, l)$ has the exact $m$-dependence as is shown in (29). So, contrary to the $\mathrm{QED}_{4}$ case, the coefficients of $\varepsilon$-expansion in the case of $\mathrm{QED}_{4,3}$ contain exactly these $\zeta_{2}$ values. However, let us note that $p_{s=2}^{(\mathrm{MV})}(m, l)=p_{s=2}^{(\mathrm{g})}(m, l)$ as it was in the $\mathrm{QED}_{4}$ case.

\section{APPENDIX B: REDUCED SCALAR QED $\mathbf{4}_{\mathbf{4} 3}$}

For completeness, we shall consider here the case of reduced scalar (spin-0) QED which is similar to reduced spinor QED that has been considered throughout the rest of the paper.

These (massless) models have the Lagrangian (in Minkowski space)

$\mathcal{L}=\left|D_{\mu} \phi\right|^{2}-\frac{1}{4} F_{\mu \nu} F^{\mu \nu}-\frac{1}{2 \xi}\left(\partial_{\mu} A^{\mu}\right)^{2}+\frac{\lambda}{4 !}\left(|\phi|^{2}\right)^{2}$

to be integrated over the appropriate volume element for the theory under consideration. As before, we only focus on the gauge-covariance of the scalar propagator.

The general expression of a scalar propagator, $S_{C}(p, \eta)$, of external momentum $p$ and gauge fixing parameter $\eta$ reads

$$
S_{C}(p, \eta)=\frac{1}{p^{2}} \sum_{m=0}^{\infty} a_{m}^{c}(\eta) A^{m}\left(\frac{\tilde{\mu}^{2}}{p^{2}}\right)^{m \varepsilon}
$$

where $a_{m}^{c}(\eta)$ are the coefficients of the loop expansion of the propagator and $\tilde{\mu}$ is the renormalization scale (14).
Proceeding in a way similar to the spinor case, the scalar propagator in another gauge $\xi$ is obtained from the following LKF transformation:

$$
S_{C}(p, \xi)=\frac{1}{p^{2}} \sum_{m=0}^{\infty} a_{m}^{c}(\xi) A^{m}\left(\frac{\tilde{\mu}^{2}}{p^{2}}\right)^{m \varepsilon}
$$

where

$$
\begin{aligned}
a_{m}^{c}(\xi)= & a_{m}^{c}(\eta) \frac{\Gamma\left(1-\varepsilon_{\mathrm{e}}-(m+1) \varepsilon\right)}{\Gamma(1+m \varepsilon)} \\
& \times \sum_{l=0}^{\infty} \frac{\Gamma(1+(m+l) \varepsilon) \Gamma^{l}(1-\varepsilon)}{l ! \Gamma\left(1-\varepsilon_{\mathrm{e}}-(m+l+1) \varepsilon\right)} \frac{(\Delta A)^{l}}{(-\varepsilon)^{l}}\left(\frac{\tilde{\mu}^{2}}{p^{2}}\right)^{l \varepsilon},
\end{aligned}
$$

which is valid for arbitrary $\varepsilon_{\mathrm{e}}$. The only difference with respect to the spinorial case is that, in the latter, there is an additional factor of $\left(1-\varepsilon_{\mathrm{e}}-(m+1) \varepsilon\right) /\left(1-\varepsilon_{\mathrm{e}}-\right.$ $(m+l+1) \varepsilon)$ as can be seen from

$$
\begin{aligned}
a_{m}(\xi)= & a_{m}(\eta) \frac{\Gamma\left(2-\varepsilon_{\mathrm{e}}-(m+1) \varepsilon\right)}{\Gamma(1+m \varepsilon)} \\
& \times \sum_{l=0}^{\infty} \frac{\Gamma(1+(m+l) \varepsilon) \Gamma^{l}(1-\varepsilon)}{l ! \Gamma\left(2-\varepsilon_{\mathrm{e}}-(m+l+1) \varepsilon\right)} \frac{(\Delta A)^{l}}{(-\varepsilon)^{l}}\left(\frac{\tilde{\mu}^{2}}{p^{2}}\right)^{l \varepsilon},
\end{aligned}
$$

which simply generalizes (16) to arbitrary $\varepsilon_{\mathrm{e}}$.

In the case of scalar $\operatorname{QED}_{4,3}\left(\varepsilon_{\mathrm{e}}=1 / 2\right)$, Eq. (B4) can then be written as

$$
a_{m}^{c}(\xi)=a_{m}^{c}(\eta) \sum_{l=0}^{\infty} \Phi_{p}(m, l, \varepsilon) \frac{(\Delta A)^{l}}{(-\varepsilon)^{l} l !}\left(\frac{\mu_{p}^{2}}{4 p^{2}}\right)^{l \varepsilon}
$$

where $\Phi_{p}(m, l, \varepsilon)$ is given by Eq. (23) for the $\overline{\mathrm{MS}}$ scale and by Eqs. (A7), (A13), and (A18) for the $g_{-}, g_{R^{-}}$, and MVscales, respectively. So, the only difference between (A1) and (B6) is in the factor $(1-2(m+1) \varepsilon) /(1-2(m+$ $l+1) \varepsilon)$ which is absent in the scalar $\mathrm{QED}_{4,3}$ case.
[1] L. D. Landau and I. M. Khalatnikov, Zh. Eksp. Teor. Fiz. 29, 89 (1955) [Sov. Phys. JETP 2, 69 (1956)]; E. S. Fradkin, Zh. Eksp. Teor. Fiz. 29, 258 (1955) [Sov. Phys. JETP 2, 361 (1956)].

[2] K. Johnson and B. Zumino, Phys. Rev. Lett. 3, 351 (1959); B. Zumino, J. Math. Phys. (N.Y.) 1, 1 (1960).
[3] S. Okubo, Nuovo Cimento 15, 949 (1960); I. BialynickiBirula, Nuovo Cimento 17, 951 (1960); H. Sonoda, Phys. Lett. B 499, 253 (2001).

[4] D. C. Curtis and M. R. Pennington, Phys. Rev. D 42, 4165 (1990); Z. h. Dong, H. J. Munczek, and C. D. Roberts, Phys. Lett. B 333, 536 (1994); A. Bashir and M. R. Pennington, 
Phys. Rev. D 50, 7679 (1994); 53, 4694 (1996); A. Bashir, A. Kizilersu, and M. R. Pennington, Phys. Rev. D 57, 1242 (1998).

[5] C. J. Burden and P. C. Tjiang, Phys. Rev. D 58, 085019 (1998); A. Bashir, A. Kizilersu, and M. R. Pennington, Phys. Rev. D 62, 085002 (2000); A. Bashir and A. Raya, Phys. Rev. D 64, 105001 (2001).

[6] S. Jia and M. R. Pennington, Phys. Lett. B 769, 146 (2017); Phys. Rev. D 94, 116004 (2016); 96, 036021 (2017).

[7] L. A. Fernandez-Rangel, A. Bashir, L. X. GutierrezGuerrero, and Y. Concha-Sanchez, Phys. Rev. D 93, 065022 (2016); N. Ahmadiniaz, A. Bashir, and C. Schubert, Phys. Rev. D 93, 045023 (2016).

[8] A. Kizilersu and M. R. Pennington, Phys. Rev. D 79, 125020 (2009).

[9] A. Bashir and A. Raya, Phys. Rev. D 66, 105005 (2002).

[10] S. Jia and M. R. Pennington, Phys. Rev. D 95, 076007 (2017).

[11] A. V. Kotikov and S. Teber, Phys. Rev. D 100, 105017 (2019).

[12] A. Ahmad, J. J. Cobos-Martínez, Y. Concha-Sánchez, and A. Raya, Phys. Rev. D 93, 094035 (2016).

[13] T. De Meerleer, D. Dudal, S. P. Sorella, P. Dall'Olio, and A. Bashir, Phys. Rev. D 97, 074017 (2018).

[14] T. De Meerleer, D. Dudal, S. P. Sorella, P. Dall'Olio, and A. Bashir, arXiv:1911.01907.

[15] D. J. Broadhurst, arXiv:hep-th/9909185.

[16] P. A. Baikov and K. G. Chetyrkin, Nucl. Phys. B837, 186 (2010).

[17] P. A. Baikov and K. G. Chetyrkin, J. High Energy Phys. 06 (2018) 141.

[18] P. A. Baikov and K. G. Chetyrkin, Proc. Sci., LL2018 (2018) 008.

[19] P. A. Baikov and K. G. Chetyrkin, J. High Energy Phys. 10 (2019) 190.

[20] S. Teber, Phys. Rev. D 86, 025005 (2012); A. V. Kotikov and S. Teber, Phys. Rev. D 87, 087701 (2013); S. Teber, Phys. Rev. D 89, 067702 (2014).

[21] A. V. Kotikov and S. Teber, Phys. Rev. D 89, 065038 (2014).

[22] S. Teber and A. V. Kotikov, Phys. Rev. D 97, 074004 (2018).

[23] P. R. Wallace, Phys. Rev. 71, 622 (1947).

[24] G. W. Semenoff, Phys. Rev. Lett. 53, 2449 (1984).

[25] K. S. Novoselov, A. K. Geim, S. V. Morozov, D. Jiang, M. I. Katsnelson, I. V. Grigorieva, S. V. Dubonos, and A. A. Firsov, Nature (London) 438, 197 (2005).

[26] M. Polini, F. Guinea, M. Lewenstein, H. C. Manoharan, and V. Pellegrini, Nat. Nanotechnol. 8, 625 (2013).

[27] M. Z. Hasan and C. L. Kane, Rev. Mod. Phys. 82, 3045 (2010).

[28] W. Pan, W. Kang, K. W. Baldwin, K. W. West, L. N. Pfeiffer, and D. C. Tsui, Nat. Phys. 13, 1168 (2017).

[29] A. V. Kotikov and S. Teber, Phys. Rev. D 94, 114010 (2016); 99, 119902(E) (2019).
[30] J. B. Marston and I. Affleck, Phys. Rev. B 39, 11538 (1989).

[31] L. B. Ioffe and A. I. Larkin, Phys. Rev. B 39, 8988 (1989).

[32] I. F. Herbut, Phys. Rev. B 66, 094504 (2002).

[33] J. González, F. Guinea, and M. A. H. Vozmediano, Nucl. Phys. B424, 595 (1994).

[34] S. Teber and A. V. Kotikov, Europhys. Lett. 107, 57001 (2014); J. High Energy Phys. 07 (2018) 082.

[35] D. Muñoz-Segovia and A. Cortijo, arXiv:1910.08081.

[36] S. Teber and A. V. Kotikov, Theor. Math. Phys. 190, 446 (2017).

[37] S. Teber and A. V. Kotikov, Theor. Math. Phys. 200, 1222 (2019).

[38] Note that Eq. (2) which was first derived in [20] was also the starting point of the analysis of [12].

[39] A. V. Kotikov and S. Teber, Phys. Part. Nucl. 50, 1 (2019).

[40] We would also like to note that in the framework of the three- and four-dimensional QED, the cases with the massive fermion propagators have been considered in Ref. [9].

[41] A. V. Kotikov and L. N. Lipatov, Nucl. Phys. B582, 19 (2000).

[42] A. V. Kotikov and L. N. Lipatov, Nucl. Phys. B661, 19 (2003); A. V. Kotikov, L. N. Lipatov, A. I. Onishchenko, and V. N. Velizhanin, Phys. Lett. B 595, 521 (2004).

[43] J. Fleischer, A. V. Kotikov, and O. L. Veretin, Nucl. Phys. B547, 343 (1999).

[44] A. V. Kotikov, L. N. Lipatov, A. Rej, M. Staudacher, and V. N. Velizhanin, J. Stat. Mech. (2007) P10003; Z. Bajnok, R. A. Janik, and T. Lukowski, Nucl. Phys. B816, 376 (2009); T. Lukowski, A. Rej, and V. N. Velizhanin, Nucl. Phys. B831, 105 (2010); C. Marboe, V. Velizhanin, and D. Volin, J. High Energy Phys. 07 (2015) 084; C. Marboe and V. Velizhanin, J. High Energy Phys. 11 (2016) 013.

[45] L. J. Dixon, I. Moult, and H. X. Zhu, Phys. Rev. D 100, 014009 (2019); J. Broedel, C. Duhr, F. Dulat, B. Penante, and L. Tancredi, J. High Energy Phys. 05 (2019) 120.

[46] D. J. Broadhurst and A. V. Kotikov, Phys. Lett. B 441, 345 (1998).

[47] Since here we are working in Euclidean space, all the factors $\left(\bar{\mu}^{2} /\left(-p^{2}\right)\right)$ in Ref. [22] should be replaced by $\left(\bar{\mu}^{2} / p^{2}\right)$.

[48] A. A. Vladimirov, Teor. Mat. Fiz. 43, 210 (1980) [Theor. Math. Phys. 43, 417 (1980)].

[49] Notice that while two-loop results are scheme independent, it is not the case of the three-loop ones which would take a different form in an alternate scheme choice.

[50] Some of these constraints at low orders read $Z_{\psi}^{(3,-3)}=$ $Z_{\psi}^{(2,-2)} Z_{\psi}^{(1,-1)}-\left(Z_{\psi}^{(1,-1)}\right)^{3} / 3$ and $Z_{\psi,}^{(3,-2)}=Z_{\psi}^{(2,-1)} Z_{\psi}^{(1,-1)}$.

[51] A. G. Grozin, Phys. Lett. B 692, 161 (2010); arXiv: 1305.4245.

[52] H. Kissler, Proc. Sci., LL2018 (2018) 032.

[53] K. G. Chetyrkin, A. L. Kataev, and F. V. Tkachov, Nucl. Phys. B174, 345 (1980). 